\title{
Experimental detection of turbulent thermal diffusion of aerosols in non-isothermal flows
}

\author{
A. Eidelman, T. Elperin, N. Kleeorin, A. Markovich, and I. Rogachevskii \\ The Pearlstone Center for Aeronautical Engineering, Studies, Department of Mechanical Engineering, The Ben-Gurion \\ University of the Negev, POB 653, Beer-Sheva 84 105, Israel
}

Received: 3 August 2005 - Revised: 16 January 2006 - Accepted: 19 January 2006 - Published: 24 April 2006

\begin{abstract}
We studied experimentally a new phenomenon of turbulent thermal diffusion of particles which can cause formation of the large-scale aerosol layers in the vicinity of the atmospheric temperature inversions. This phenomenon was detected experimentally in oscillating grids turbulence in air flow. Three measurement techniques were used to study turbulent thermal diffusion in strongly inhomogeneous temperature fields, namely Particle Image Velocimetry to determine the turbulent velocity field, an image processing technique to determine the spatial distribution of aerosols, and an array of thermocouples for the temperature field. Experiments are presented for both, stably and unstably stratified fluid flows, by using both directions of the imposed mean vertical temperature gradient. We demonstrated that even in strongly inhomogeneous temperature fields particles in turbulent fluid flow accumulate at the regions with minimum of mean temperature of surrounding fluids due to the phenomenon of turbulent thermal diffusion.
\end{abstract}

\section{Introduction}

Various aspects of turbulent diffusion of aerosols have been investigated analytically, numerically and experimentally in the past (see, e.g., Csanady, 1980; Maxey, 1987; Fessler et al., 1994; Blackadar, 1997; Warhaft, 2000; Ott and Mann, 2000; Aliseda et al., 2002). In particular, different approaches and methods were used to determine the coefficient of turbulent diffusion. Nevertheless, certain important features of turbulent transport of aerosols in non-isothermal flows have been found only recently. In particular, turbulent thermal diffusion was recently predicted theoretically by Elperin et al. (1996, 1997). The essence of this phenomenon is the appearance of a nondiffusive mean flux of aerosols in

Correspondence to: T. Elperin

(elperin@bgu.ac.il) the direction of the turbulent heat flux which would cause formation of large-scale inhomogeneities in the spatial distribution of aerosols in regions of minimum mean temperature of the surrounding fluid. For large Reynolds and Peclet numbers the turbulent thermal diffusion is much stronger than the molecular thermal diffusion (see, e.g., Landau and Lifshits, 1987).

The mechanism of turbulent thermal diffusion for $\rho_{p} \gg \rho$ is as follows, where $\rho_{p}$ is the material density of particles and $\rho$ is the density of the fluid. The inertia causes particles inside the turbulent eddies to drift out to the boundary regions between eddies (i.e., regions with low vorticity or high strain rate and maximum fluid pressure) and accumulate there. Similarly, there is an outflow of particles from regions with minimum fluid pressure. In homogeneous and isotropic turbulence without large-scale external gradients of temperature, a drift from regions with increased (decreased) concentration of particles by a turbulent flow of fluid is equiprobable in all directions, and pressure (temperature) of the surrounding fluid is not correlated with the turbulent velocity field. Thus, there exists only turbulent diffusion of particles. The situation drastically changes in a turbulent fluid flow with a mean temperature gradient, whereby the turbulent heat flux $\left\langle\mathbf{u}^{\prime} \theta^{\prime}\right\rangle$ is not zero, i.e., fluctuations of fluid temperature $\theta^{\prime}$ and velocity $\mathbf{u}^{\prime}$ of the fluid are correlated. Fluctuations of temperature cause pressure fluctuations, which result in fluctuations of the number density of particles. Increase (decrease) of the pressure of the surrounding fluid is accompanied by accumulation (outflow) of the particles. Therefore, the direction of mean flux of particles coincides with that of the turbulent heat flux, i.e. $\left\langle\mathbf{u}^{\prime} n^{\prime}\right\rangle \propto\left\langle\mathbf{u}^{\prime} \theta^{\prime}\right\rangle \propto-\nabla T$, where $T$ is the mean fluid temperature and $n^{\prime}$ are the fluctuations of particle number density. The mean flux of particles is directed to the minimum mean temperature, and the particles are accumulated in this region (see Elperin et al., 1996, 1997).

In order to demonstrate that the directions of the mean flux of particles and the turbulent heat flux coincide, let us

Published by Copernicus GmbH on behalf of the European Geosciences Union and the American Geophysical Union. 


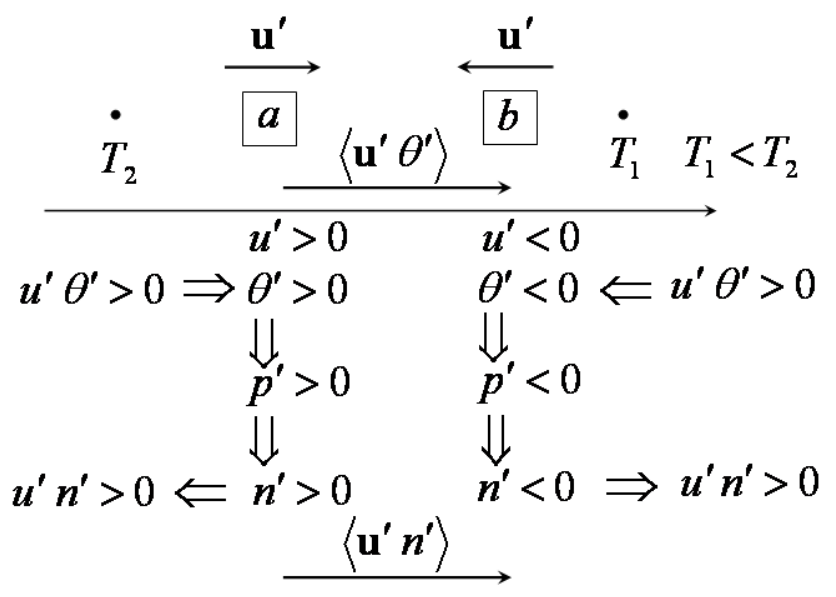

Fig. 1. Mechanism of turbulent thermal diffusion.

assume that the mean temperature $T_{2}$ at point 2 is larger than the mean temperature $T_{1}$ at point 1 (see Fig. 1). Consider two small control volumes " $a$ " and " $b$ " located between these two points (see Fig. 1), and let the direction of the local turbulent velocity at the control volume " $a$ " at some instant be the same as the direction of the turbulent heat flux $\left\langle\mathbf{u}^{\prime} \theta^{\prime}\right\rangle$ (i.e., to the point 1$)$. Let the local turbulent velocity at the control volume " $b$ " be directed at this instant opposite to the turbulent heat flux (i.e., to the point 2). In a fluid flow with an imposed mean temperature gradient, pressure $p^{\prime}$ and velocity $\mathbf{u}^{\prime}$ fluctuations are correlated, and regions with a higher level of pressure fluctuations have higher temperature and velocity fluctuations. Thus, the fluctuations of the temperature $\theta^{\prime}$ and pressure $p^{\prime}$ at the control volumes " $a$ " are positive, and at the control volume " $b$ " they are negative. The fluctuations of the particle number density $n^{\prime}$ are positive in the control volume " $a$ " (because particles are locally accumulated in the vicinity of the maximum of pressure fluctuations), and they are negative at the control volume " $b$ " (because there is an outflow of particles from regions with a low pressure). The mean flux of particles $\left\langle\mathbf{u}^{\prime} n^{\prime}\right\rangle$ is positive in the control volume " $a$ " (i.e., it is directed to the point 1), and it is also positive at the control volume " $b$ " (because both fluctuations of velocity and number density of particles are negative at the control volume " $b$ "). Therefore, the mean flux of particles $\left\langle\mathbf{u}^{\prime} n^{\prime}\right\rangle$ is directed, as is the turbulent heat flux $\left\langle\mathbf{u}^{\prime} \theta^{\prime}\right\rangle$, towards the point 1 .

It was shown by Elperin et al. $(1996,1997)$ that the mean number density of particles $N$ in a turbulent fluid flow is governed by the following equations

$$
\begin{aligned}
& \frac{\partial N}{\partial t}+\operatorname{div}\left[N\left(\mathbf{V}+\mathbf{W}+\mathbf{V}_{\mathrm{eff}}\right)-D_{T} \nabla N\right]=0, \\
& \mathbf{V}_{\mathrm{eff}}=-\tau\left\langle\mathbf{u}^{\prime} \operatorname{div} \mathbf{u}^{\prime}\right\rangle=-D_{T}(1+\kappa) \frac{\nabla T}{T},
\end{aligned}
$$

where $D_{T}=(\tau / 3)\left\langle\mathbf{u}^{\prime 2}\right\rangle$ is the turbulent diffusion coefficient, $\tau$ is the correlation time of the turbulent velocity field, $\mathbf{V}$ is the mean velocity, $\mathbf{W}=\tau_{p} \mathbf{g}$ is the terminal fall velocity, $\mathbf{g}$ is the acceleration due to gravity, $\tau_{p}$ is the characteristic time of coupling between the particle and atmospheric fluid (Stokes time), the coefficient $\kappa$ depends on a particle size, the Reynolds number and the mean fluid temperature. For noninertial particles the coefficient $\kappa=0$. In Eq. (1) we did not take into account a small molecular mean flux of particles caused by molecular (Brownian) diffusion and molecular thermal diffusion (molecular thermophoresis). The term $-D_{T} \nabla N$ in the square brackets of Eq. (1) describes the turbulent diffusion of particles, while the term $N \mathbf{V}_{\text {eff }}$ determines the additional turbulent flux of particles caused by turbulent thermal diffusion. Equations (1) and (2) were derived using different methods by Elperin et al. (1996, 1997, 2001), Pandya and Mashayek (2002) and Reeks (2005).

First experiments in oscillating grids turbulence that demonstrated the phenomenon of turbulent thermal diffusion, were discussed by Eidelman et al. (2004) and Buchholz et al. (2004). Later, the phenomenon of turbulent thermal diffusion was observed in a multi-fan turbulence generator (Eidelman et al., 2006). Observation time in these devices is almost unlimited due to the small mean velocity of the flow. The main goal of this paper is to study experimentally the phenomenon of turbulent thermal diffusion in strongly inhomogeneous temperature fields.

Three measurement techniques were used to study turbulent thermal diffusion in strongly inhomogeneous temperature fields, namely Particle Image Velocimetry for the turbulent velocity field, an image processing technique to determine the spatial distribution of aerosols, and an array of thermocouples for the temperature field. Experiments are presented for both, stably and unstably stratified fluid flows, by using both directions of the imposed mean vertical temperature gradient. We demonstrated that even in strongly inhomogeneous temperature fields particles in non-isothermal turbulent fluid flow accumulate at the regions with minimum of mean temperature of surrounding fluids due to the phenomenon of turbulent thermal diffusion.

\section{Turbulent thermal diffusion in atmospheric flow}

Turbulent thermal diffusion in non-isothermal flows can be important in atmospheric phenomena (atmospheric aerosols, transport of pollutants and smog formation, see, e.g., Twomey, 1977; Seinfeld, 1986; Jaenicke, 1987; Flagan and Seinfeld, 1988; Telford, 1996; Shaw, 2003) and industrial turbulent flows (internal combustion engines, see, e.g., Heywood, 1988; Kamimoto and Kobayashi, 1991; Glassman, 1996; Crowe et al., 1998; Borman and Ragland, 1999). The effect of turbulent thermal diffusion results in the formation of large-scale inhomogeneities in the spatial distribution of aerosols (see Elperin et al., 2000a, b). Localization of these inhomogeneities is correlated with the temperature inversion regions. The effective velocity of aerosols due to turbulent 
Table 1. The ratio $\left|\mathbf{V}_{\text {eff }} / W\right|$ for the atmospheric flows with Tinversions.

\begin{tabular}{lccc}
\hline$a_{*}$ & $1 \mathrm{~K} / 100 \mathrm{~m}$ & $1 \mathrm{~K} / 200 \mathrm{~m}$ & $1 \mathrm{~K} / 300 \mathrm{~m}$ \\
\hline $1 \mu \mathrm{m}$ & 13 & 6.5 & 4.33 \\
$5 \mu \mathrm{m}$ & 3.4 & 1.7 & 1.13 \\
$10-20 \mu \mathrm{m}$ & 3 & 1.5 & 1 \\
$30 \mu \mathrm{m}$ & 2.7 & 1.35 & 0.9 \\
\hline
\end{tabular}

thermal diffusion in the atmospheric turbulent flows was derived by Elperin et al. (2000a, b) to be:

$\mathbf{V}_{\text {eff }}=-W A\left(\operatorname{Re}, a_{*}\right) \Lambda_{P} \ln (\operatorname{Re}) \frac{\nabla T}{T}$,

where $a_{*}$ is the particle radius, $\Lambda_{P}=|\nabla P / P|^{-1}, \quad P$ is the mean fluid pressure, $\operatorname{Re}=l u / v$ is the Reynolds number, $l$ is the maximum scale of atmospheric turbulent motions, $u$ is the characteristic velocity in this scale, and $v$ is the kinematic viscosity. The function $A\left(\operatorname{Re}, a_{*}\right)=1$ for $a_{*}<a_{\mathrm{cr}}$, and $A\left(\operatorname{Re}, a_{*}\right)=1-3 \ln \left(a_{*} / a_{\mathrm{cr}}\right) / \ln (\operatorname{Re})$ with $a_{*} \geq a_{\mathrm{cr}}$, where $a_{\mathrm{cr}}=\eta\left(\rho / \rho_{p}\right)^{1 / 2}$ and $\eta=l \mathrm{Re}^{-3 / 4}$ is the viscous (Kolmogorov) scale of turbulent motions. For atmospheric turbulent flow, $a_{\mathrm{cr}} \sim 20 \mu \mathrm{m}$, where $\mathrm{Re}=10^{7}$, $l=100 \mathrm{~m}$ and $\rho_{p}=10^{3} \mathrm{~kg} / \mathrm{m}^{3}$.

The effective velocity of aerosols, $\mathbf{V}_{\text {eff }}$, determined by Eq. (3) can cause formation of inhomogeneities in the spatial distribution of aerosols. Equation (3) for the effective velocity of aerosols provides a local parameterization of these turbulence effects which can be directly incorporated to existing atmospheric numerical models. The ratio $\left|\mathbf{V}_{\text {eff }} / W\right|$ can be estimated from Eq. (3) as:

$\left|\frac{\mathbf{V}_{\text {eff }}}{W}\right| \sim \frac{\Lambda_{P}}{\Lambda_{T}} \frac{\delta T}{T_{*}} \ln (\mathrm{Re})$,

where $\delta T$ is the temperature difference in the scale $\Lambda_{T}=|\nabla T / T|^{-1}$, and $T_{*}$ is the characteristic temperature. The ratio $\left|\mathbf{V}_{\text {eff }} / W\right|$ determined by Eq. (3) for the atmospheric turbulent flows with temperature inversion and different aerosol sizes is given in Table 1. This demonstrates that the additional velocity $\mathbf{V}_{\text {eff }}$ of aerosols can be larger than the terminal fall velocity $W$. In the atmosphere without temperature inversion, the effective particle velocity is directed opposite to the terminal fall velocity, and thus the effective particle velocity decreases the effective sedimentation velocity by 10 to 30 percent. In the atmosphere with a temperature inversion, the effective particle velocity $\mathbf{V}_{\text {eff }}$ is directed to the temperature minimum and it results in accumulation of particles in the vicinity of the temperature inversion.

Therefore, the additional turbulent nondiffusive flux of particles due to the effective velocity $\mathbf{V}_{\text {eff }}$ results in formation of inhomogeneities of aerosols distribution. The characteristic time of formation of inhomogeneities of aerosols is $\tau_{a} \sim \Lambda_{T} /\left|V_{\text {eff }}-W\right|$ and the formation of inhomogeneities is

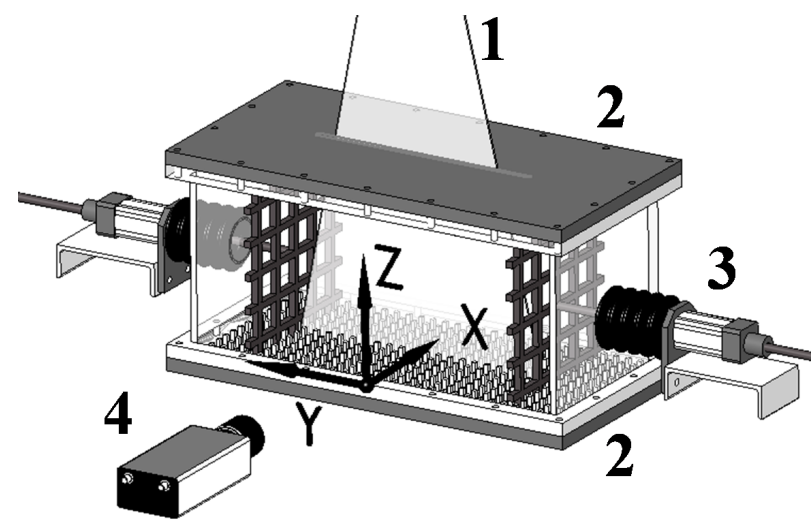

Fig. 2. Scheme of the experimental set-up: laser light sheet (1); test section of the oscillating grids turbulence generator with the heat exchangers (2) at the top and bottom walls; rods driven by the speed-controlled motors (3); digital CCD camera (4).

possible when $V_{\text {eff }}>W$. Therefore, it is important to take into account the additional turbulent nondiffusive flux of aerosols due to the effective velocity $\mathbf{V}_{\text {eff }}$ in atmospheric phenomena (e.g., atmospheric aerosols, cloud formation and smog formation). Observations of the vertical distributions of aerosols in the atmosphere show that maximum concentrations can occur within temperature inversion layers (see, e.g., Blackadar, 1997; Seinfeld, 1986; Jaenicke, 1987). The characteristic parameters of the atmospheric turbulent boundary layer are: maximum scale of turbulent flow $l \sim 10-100 \mathrm{~m}$; velocity in the scale $l: u \sim 0.3-1 \mathrm{~m} / \mathrm{s}$; Reynolds number $\operatorname{Re} \sim 10^{6}-10^{7}$ (Blackadar, 1997). Therefore, for particles with material density $\rho_{p} \sim(1-2) \times 10^{3} \mathrm{~kg} / \mathrm{m}^{3}$ and radius $a_{*}=20 \mu \mathrm{m}$ the characteristic time of formation of inhomogeneities is of the order of $1 \mathrm{~h}$ for a temperature gradient of $1 \mathrm{~K} / 100 \mathrm{~m}$ and $2 \mathrm{~h}$ for a temperature gradient of $1 \mathrm{~K} / 200 \mathrm{~m}$.

\section{Experimental set-up}

The experiments were carried out in a turbulence generated by oscillating grids in air. The test section of the oscillating grids turbulence generator was constructed as a rectangular chamber of dimensions $29 \times 58 \times 29 \mathrm{~cm}$ (see Fig. 2). Pairs of vertically oriented grids with bars arranged in a square array (with a mesh size $5 \mathrm{~cm}$ ) are attached to the right and left horizontal rods. The grids are positioned at a distance of two grid meshes from the chamber walls parallel to them. Both grids are operated at the same amplitude of $61 \mathrm{~mm}$, at a random phase and at the same frequency varied in the range from $2.2 \mathrm{~Hz}$ to $16.5 \mathrm{~Hz}$. Here we use the following system of coordinates: $Z$ is the vertical axis, the $Y$-axis is perpendicular to the grids and the $X Z$-plane is parallel to the grids.

A mean temperature gradient in the turbulent flow was formed with two aluminium heat exchangers attached to the bottom and top walls of the chamber. We performed 
experiments with two directions of the mean temperature gradient, for stably stratified fluid flow (the cold bottom and hot top walls of the chamber) and for unstably stratified fluid flow (the heated bottom and cold top walls of the chamber). In order to improve heat transfer in the boundary layers at the walls we used a heat exchanger with rectangular fins $(3 \times 3 \times 15 \mathrm{~mm}$, see Fig. 2$)$ which allowed us to form a mean temperature gradient $118 \mathrm{~K} / \mathrm{m}$ for the stably stratified flow and $115 \mathrm{~K} / \mathrm{m}$ for the unstably stratified flow at a mean temperature of about $310 \mathrm{~K}$.

The temperature field was measured with a temperature probe equipped with twelve E-thermocouples (with the diameter of $0.13 \mathrm{~mm}$ and the sensitivity of $40 \mu \mathrm{V} / \mathrm{K}$ ) attached to a vertical rod with a diameter $4 \mathrm{~mm}$. The spacing between thermocouples along the rod was $22 \mathrm{~mm}$. Each thermocouple was inserted into a $1 \mathrm{~mm}$ diameter and $45 \mathrm{~mm}$ long case. A tip of a thermocouple protruded at the length of $15 \mathrm{~mm}$ out of the case. The mean temperature was measured for 33 rod positions with $15 \mathrm{~mm}$ intervals in the horizontal direction, i.e., at 396 locations in a flow. The exact position of each thermocouple was measured using images captured with the optical system employed in PIV measurements. A sequence of 1024 temperature readings (each reading was averaged over 15 instantaneous measurements) for every thermocouple at every rod position was recorded and processed using the developed software based on LabView 7.0. Temperature maps and isothermal lines were plotted using MathLab7.

The turbulent velocity field was measured using a digital Particle Image Velocimetry (PIV) system with LaVision Flow Master III (see, e.g., Adrian, 1991; Raffel et al., 1998; Westerweel, 2000). A double-pulsed light sheet is provided by a Nd-YAG laser (Continuum Surelite $2 \times 170 \mathrm{~mJ}$ ). Light sheet optics comprised spherical and cylindrical Galilei telescopes with tuneable divergence and adjustable focus length. We employed a progressive-scan 12 Bit digital CCD camera (pixels with a size $6.7 \mu \mathrm{m} \times 6.7 \mu \mathrm{m}$ each) with dual frame technique for cross-correlation processing of captured images. The tracer used for PIV measurements was incense smoke with sub-micron particles (with $\rho_{p} / \rho \sim 10^{3}$ ), which was produced by high temperature sublimation of solid incense particles. Analysis of smoke particles using a microscope (Nikon, Epiphot with an amplification 560) and PM300 portable laser particulate analyzer showed that these particles have an approximately spherical shape with a mean diameter of $0.7 \mu \mathrm{m}$.

We determined the mean and the r.m.s. velocities, twopoint correlation functions and an integral scale of turbulence from the measured velocity fields. Series of 130 pairs of images, acquired with a frequency of $4 \mathrm{~Hz}$, were stored for calculating velocity maps and for ensemble and spatial averaging of turbulence characteristics. The center of the measurement region coincides with the center of the chamber. We measured velocity for flow areas from $60 \times 60 \mathrm{~mm}^{2}$ up to $212 \times 212 \mathrm{~mm}^{2}$ with a spatial resolution of $1024 \times 1024$ pixels. This corresponds to a spatial resolution from $58 \mu \mathrm{m} /$ pixel up to $207 \mu \mathrm{m} /$ pixel. These measurement regions were analyzed with interrogation windows of $32 \times 32$ or $16 \times 16$ pixels, respectively. In every interrogation window a velocity vector was determined from which velocity maps comprising $32 \times 32$ or $64 \times 64$ vectors were constructed. The mean and r.m.s. velocities for every point of a velocity map (1024 points) were calculated by averaging over 130 independent maps, and then they were averaged over 1024 points. The two-point correlation functions of the velocity field were calculated for every point of the central part of the velocity map (with $16 \times 16$ vectors) by averaging over 130 independent velocity maps, and then they were averaged over 256 points. An integral scale $l$ of turbulence was determined from the two-point correlation functions of the velocity field.

Particle spatial distribution was determined using digital Particle Image Velocimetry (PIV) system. In particular, the effect of Mie light scattering by particles was used to determine the particle spatial distribution in the flow (see, e.g., Guibert et al., 2001). In the experiments we probed the central $212 \times 212 \mathrm{~mm}^{2}$ region in the chamber. The mean intensity of scattered light was determined in $32 \times 16$ interrogation windows with the size $32 \times 64$ pixels. The vertical distribution of the intensity of the scattered light was determined in 16 vertical strips composed of 32 interrogation windows. The light radiation energy flux scattered by small particles is $E_{S} \propto E_{0} \Psi\left(\pi d_{p} / \lambda ; a_{0} ; N\right)$, where $E_{0} \propto \pi d_{p}^{2} / 4$ is the energy flux incident at the particle, $d_{p}$ is the particle diameter, $\lambda$ is the wavelength, $a_{0}$ is the index of refraction and $\Psi$ is the scattering function. For wavelengths $\lambda$ which are larger than the particle perimeter $\left(\lambda>\pi d_{p}\right)$, the function $\Psi$ is given by Rayleigh's law, $\Psi \propto d_{p}^{4}$. If the wavelength is small, the function $\Psi$ tends to be independent of $d_{p}$ and $\lambda$. In the general case the function $\Psi$ is given by Mie's equations (see, e.g., Bohren and Huffman, 1983, Chapter 4). The scattered light energy flux incident on the CCD camera probe (producing proportional charge in every CCD pixel) is proportional to the particle number density $N$, i.e., $E_{s} \propto E_{0} N\left(\pi d_{p}^{2} / 4\right)$. The probability density function of the particle size (measured with the PM300 particulate analyzer) was independent of the location in the flow. Indeed, since the number density of particles is small, so that they are about $1 \mathrm{~mm}$ apart, it can be safely assumed that a change in particle number density $N$ does not affect their size distribution. Consequently, the ratio of the scattered radiation fluxes at two locations in the flow and at the image measured with the CCD camera is equal to the ratio of the particle mean number densities at these two locations. Measurements performed using different concentration of the incense smoke showed that the distribution of the average scattered light intensity over a vertical coordinate was independent of the mean particle number density in the isothermal flow.

In order to characterize the spatial distribution of particle number density $N \propto E^{T} / E$ in the non-isothermal flow, the distribution of the scattered light intensity $E$ measured in the isothermal case was used for the normalization of 
the scattered light intensity $E^{T}$ obtained in a non-isothermal flow under the same conditions. The scattered light intensities $E^{T}$ and $E$ in each experiment were normalized by corresponding scattered light intensities averaged over the vertical coordinate. Mie scattering is not affected by temperature change because it depends on the electric permittivity of particles, the particle size and the laser light wave length. The temperature effect on these characteristics is negligibly small.

\section{Experimental results}

All experiments were conducted at the same temperature difference between the top and bottom walls $\Delta T=50 \mathrm{~K}$ (e.g., for stably stratified flow, the bottom wall temperature was $283 \mathrm{~K}$, the top wall temperature was $333 \mathrm{~K}$, and vice versa for unstably stratified flow). For stably stratified flow, the frequency of the grid oscillations varied from $f=4.5 \mathrm{~Hz}$ to $f=16.5 \mathrm{~Hz}$, and for unstably stratified flow, the frequency of the grid oscillations varied from $f=2.2 \mathrm{~Hz}$ to $f=16.4 \mathrm{~Hz}$. Our PIV measurements confirmed earlier results (Turner, 1968, 1973; Thompson and Turner, 1975; Hopfinger and Toly, 1976; Kit et al., 1997; Sánchez and Redondo, 1998; Medina et al., 2001) for the dependence of varies characteristics of the turbulent velocity field on the parameters of the oscillating grid turbulence generator in water flow experiments. In particular, the integral scale of turbulence is proportional to the distance from a grid, $l \propto Y$, and the r.m.s. velocity is $\sqrt{\left\langle\mathbf{u}^{\prime 2}\right\rangle} \propto f Y^{-1}$ (see for details, Buchholz et al., 2004, for air flow experiments). This implies that Reynolds numbers and the turbulent diffusion coefficient in the core of the flow are independent of the distances from the grid. The turbulent flow parameters in the oscillating grids turbulence generator are as follows: the r.m.s. velocity is $\sqrt{\left\langle\mathbf{u}^{\prime 2}\right\rangle}=3.6-14 \mathrm{~cm} / \mathrm{s}$ depending on the frequency of grid oscillations, the integral scale of turbulence is $l=1.6-2.3 \mathrm{~cm}$, the Kolmogorov length scale is $\eta=380-660 \mu \mathrm{m}$.

The dependence of the r.m.s. velocities: $\sqrt{\left\langle u_{y}^{\prime 2}\right\rangle}$ and $\sqrt{\left\langle u_{z}^{\prime 2}\right\rangle}$ versus a frequency $f$ of the two-grids-oscillations system for the stably stratified turbulent flow at the temperature difference between the top and bottom walls $\Delta T=50 \mathrm{~K}$ is shown in Fig. 3. There is a slight difference in the velocity components (about 9\%) caused by an anisotropy of forcing of the grid oscillating turbulence. The r.m.s. velocity distributions, $u(y, z)=\sqrt{\left\langle\mathbf{u}^{\prime 2}\right\rangle}$, for the unstably stratified turbulent flow generated by two grids oscillating with the frequencies $f=2.2 \mathrm{~Hz}$ and $f=10.4 \mathrm{~Hz}$ are shown in Fig. 4. This demonstrates that inhomogeneity of turbulence in the core of fluid flow is weak even for the low frequency forcing. We found a weak mean flow in the form of two large toroidal structures parallel and adjacent to the grids. The interaction of these structures results in a symmetric mean flow that is sensitive

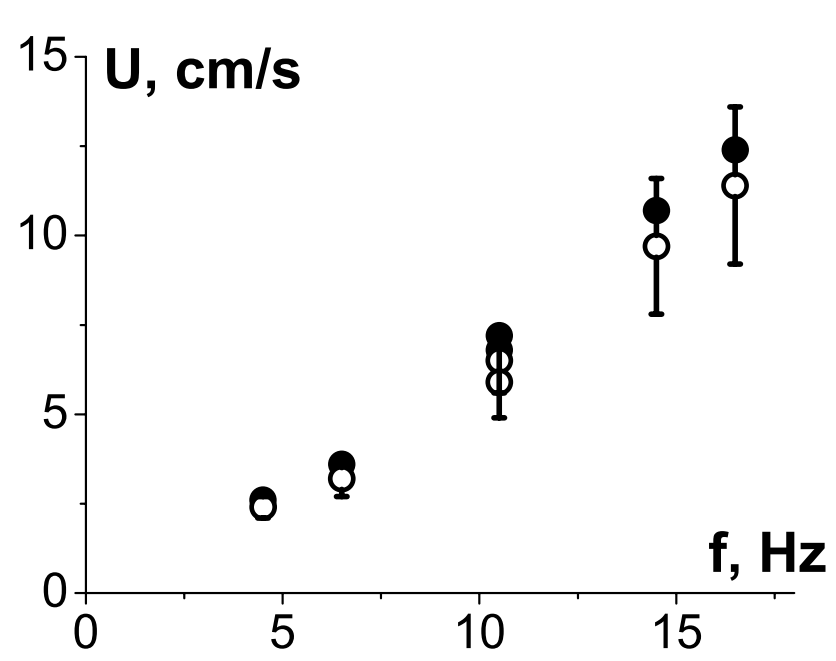

Fig. 3. Dependence of the r.m.s. velocities: $\sqrt{\left\langle u_{y}^{\prime}{ }^{2}\right\rangle}$ (unfilled circles) and $\sqrt{\left\langle u_{z}^{\prime 2}\right\rangle}$ (filled circles) versus frequency $f$ of the twogrids-oscillations for the stably stratified turbulent flow at the temperature difference between the top and bottom walls $\Delta T=50 \mathrm{~K}$.

to the parameters of grids adjustment. We studied the parameters that affect the mean flow, e.g., the grids distance to the walls of the chamber, the angles of the grids planes with the axes of their oscillation. This study allowed us to expand the central region with homogeneous turbulence by inserting partitions behind the grids. The measured r.m.s. velocity was 5 times higher than the characteristic mean velocity in the core of the flow.

In Figs. 5-7 we plotted the measured mean temperature fields and the particle number density distributions in different flow fields. In particular, Fig. 5 corresponds to the stably stratified turbulent fluid flow (the cold bottom and hot top walls of the chamber). Figures 6-7 are plotted for the unstably stratified fluid flow (the hot bottom and cold top walls of the chamber) generated by two grids oscillating with the frequencies, $f=10.5 \mathrm{~Hz}$ (Fig. 6) and $f=2.2 \mathrm{~Hz}$ (Fig. 7). In Figs. 5-7 the mean particle number density is normalized by its maximum value in every experiment. This is the reason that the particle number density is less than unity. In Figs. 57 the mean temperature is shown in degrees of C. Figures 57 demonstrate the redistribution of particles in the turbulent flow with the mean temperature gradient, so that particles are accumulated in regions with minimum mean temperature, and there is outflow of particles from the regions where the mean temperature is larger.

\section{Discussion and conclusions}

Now let us analyze the obtained experimental results. In the unstably stratified flow there are two sources of turbulence, the external mechanical forcing produced by oscillating grids 

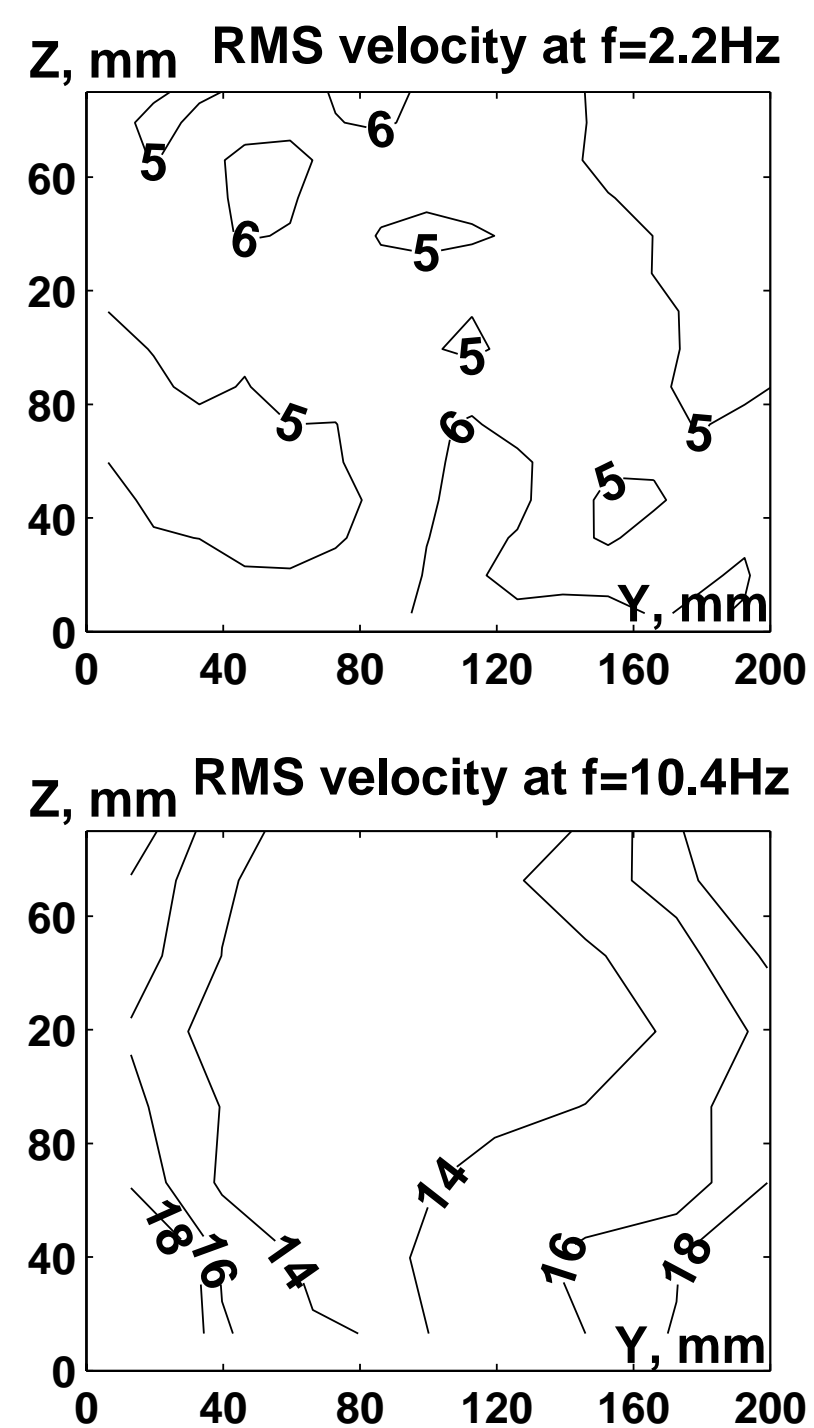

Fig. 4. The r.m.s. velocity distributions, $u(y, z)=\sqrt{\left\langle\mathbf{u}^{\prime 2}\right\rangle}$, for the unstably stratified turbulent flow generated by two grids oscillating with the frequencies $f=2.2 \mathrm{~Hz}$ and $f=10.4 \mathrm{~Hz}$.

and the buoyancy effect. Figures 6 and 7 show the results of different experiments where the frequencies of grid oscillations differ by a factor of 5 . Figure 7 corresponds to the experiment with much smaller frequency of grid oscillations, whereby the buoyancy effect plays a very important role and causes a strong change in spatial distribution of the mean temperature. The temperature field is qualitatively different at low frequency of grid oscillations. Here the vertical gradient of the mean temperature in the central part of the chamber becomes much smaller while a horizontal gradient of the mean temperature becomes of the same order of magnitude as the vertical gradient of the mean temperature. The distribution of the mean temperature in this case is close to temperature distribution in turbulent convection in

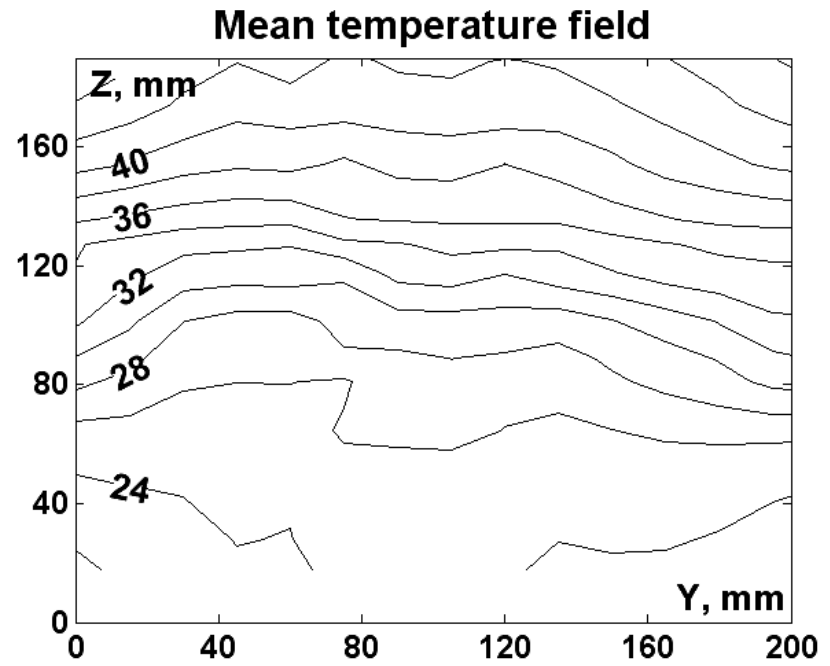

Particle number density distribution

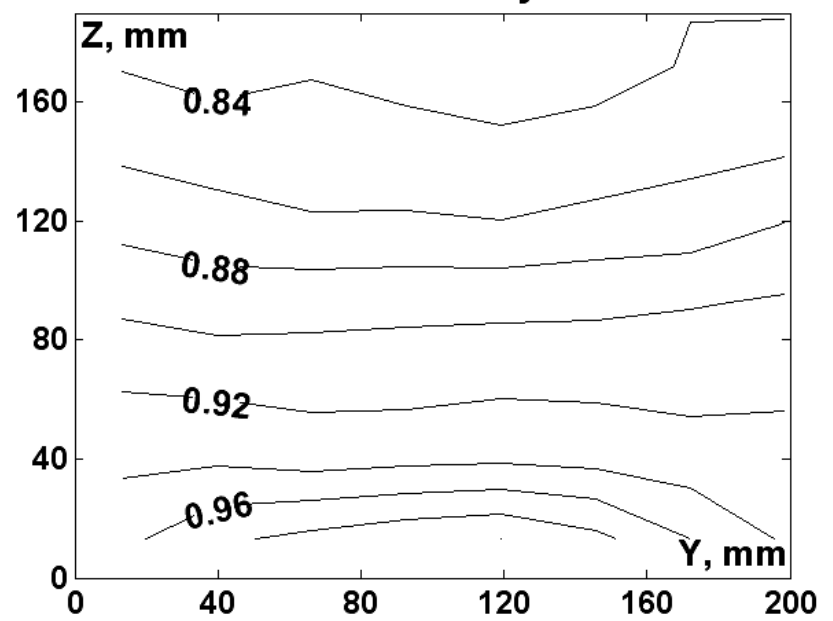

Fig. 5. Mean temperature field and particle number density distribution for the stably stratified turbulent flow generated by two grids oscillating with the frequency $f=10.5 \mathrm{~Hz}$. The mean temperature is shown in degrees of $\mathrm{C}$.

the presence of large-scale circulation. Note that the largescale circulation in turbulent convection arises at very small frequency of grid oscillations. Therefore, the spatial distribution of the mean temperature strongly depends on the ratio of the buoyancy force to the external forcing due to oscillating grids. Nevertheless, even in this three-dimensional mean temperature field, the locations of regions with the minimum mean temperature are strongly correlated with the locations of the regions with the maximum particle number density due to the phenomenon of turbulent thermal diffusion.

In these experiments the contribution of the mean flow to the spatial distribution of particles is negligibly small. In particular, the normalized distribution of the scattered light intensity measured in the different vertical strips in the flow in the regions where the mean flow velocity and coefficient of 


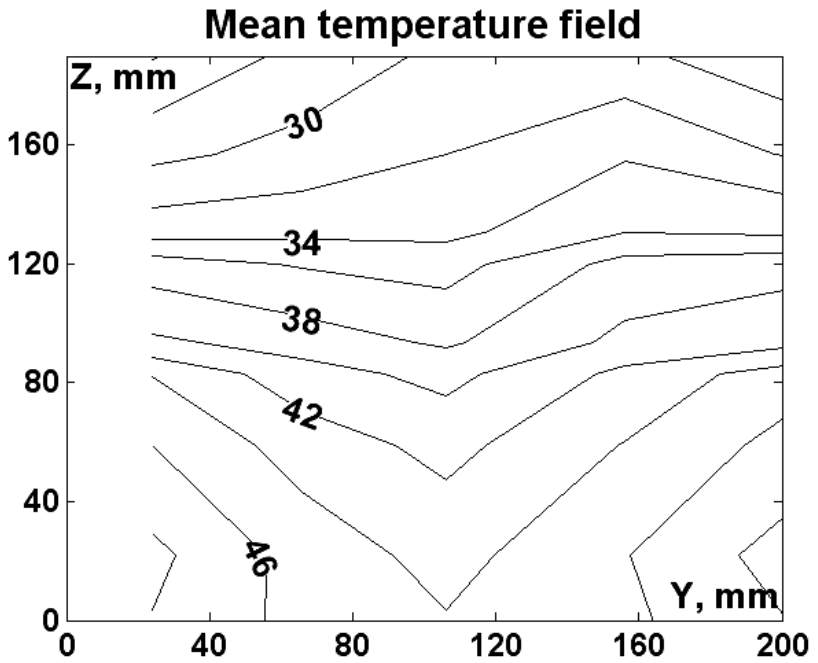

Particle number density distribution

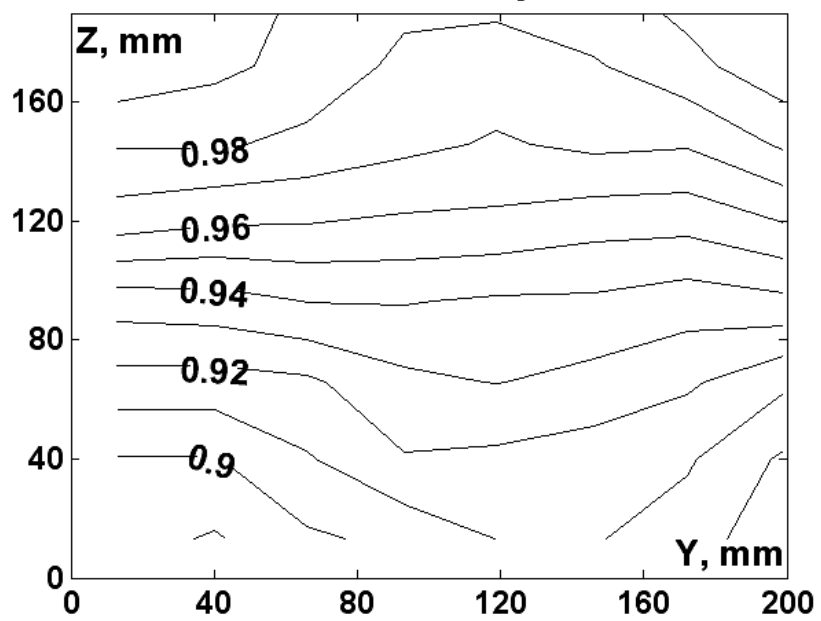

Fig. 6. Mean temperature field and particle number density distribution for the unstably stratified turbulent flow generated by two grids oscillating with the frequency $f=10.5 \mathrm{~Hz}$. The mean temperature is shown in degrees of $\mathrm{C}$.

turbulent diffusion vary strongly are practically identical (the difference is about $1 \%$ ). The number density of particles in our experiments was of the order of $10^{10}$ particles per $\mathrm{m}^{3}$, so that their collision rate is negligibly small. The effect of the gravitational settling of small particles $(0.5-1 \mu \mathrm{m})$ is negligibly small since the terminal fall velocity of these particles is less than $0.01 \mathrm{~cm} / \mathrm{s}$.

The turbulent thermal diffusion ratio, $\alpha$, was determined in the experiments in oscillating grids turbulence (Eidelman et al., 2004; Buchholz et al., 2004) and in multi-fan turbulence (Eidelman et al., 2006). The turbulent thermal diffusion ratio was introduced using the steady-state solution of Eq. (1): $\nabla N / N=-\alpha \nabla T / T$, which yields $N / N_{0}=1-\alpha\left(T-T_{0}\right) / T_{0}$, where $T_{0}$ is the reference temperature, $N_{0}=N\left(T=T_{0}\right)$ and $\alpha=1+\kappa$. For example, Fig. 8 shows the normalized particle
Mean temperature field

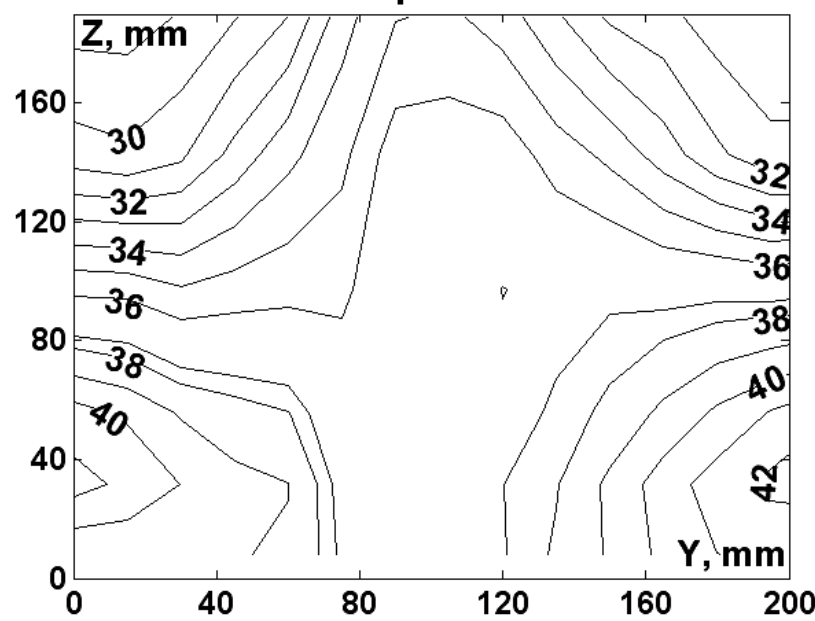

Particle number density distribution

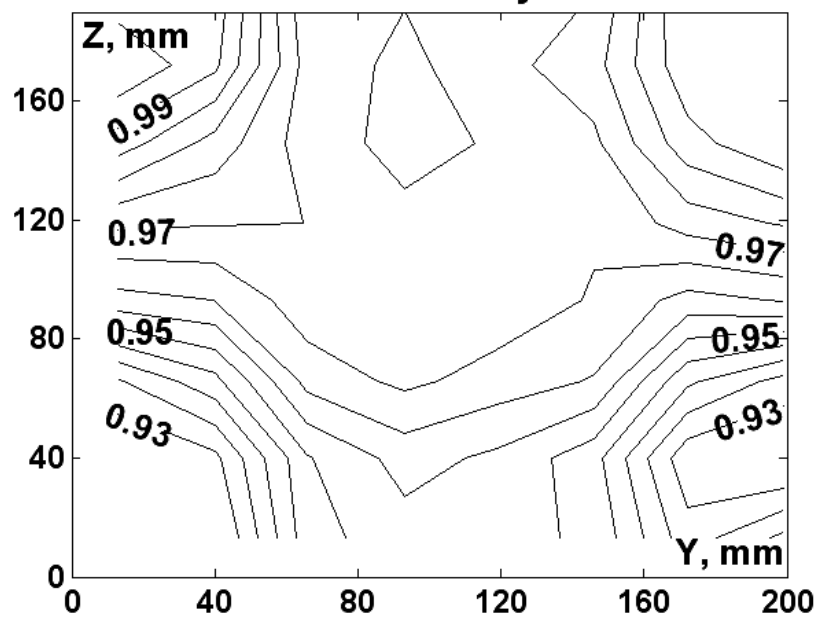

Fig. 7. Mean temperature field and particle number density distribution for the unstably stratified turbulent flow generated by two grids oscillating with the frequency $f=2.2 \mathrm{~Hz}$. The mean temperature is shown in degrees of $\mathrm{C}$.

number density $N_{z} \equiv N / N_{0}$ versus the normalized temperature difference $T_{z} \equiv\left(T-T_{0}\right) / T_{0}$ for stably and unstably stratified flows. If we do not take into account the term $N \mathbf{V}_{\text {eff }}$ in Eq. (1), then the steady-state solution of Eq. (1) is $N=N_{0}=$ const and $\alpha=0$. However, in the experiments with oscillating grids turbulence (Eidelman et al., 2004; Buchholz et al., 2004), the turbulent thermal diffusion ratio varied from $\alpha=1.29$ to $\alpha=1.87$ depending on the frequencies of the grid oscillations, and in the experiments with the multi-fan turbulence (Eidelman et al., 2006), $\alpha=2.68$. The latter value of $\alpha$ is larger than that obtained in the experiments in oscillating grids turbulence, where the Reynolds numbers were smaller than that achieved in the multi-fan turbulence generator. Therefore, all these experiments demonstrated existence of the phenomenon of turbulent thermal diffusion predicted theoretically by Elperin et al. $(1996,1997)$. 


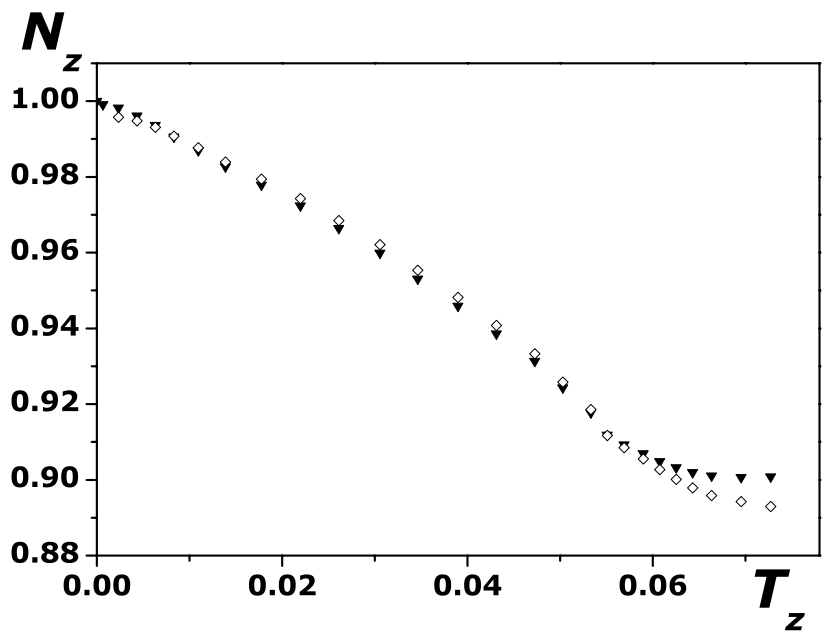

Fig. 8. Normalized particle number density $N_{z}$ versus normalized temperature difference $T_{z}$. The frequency of grid oscillations is $f=10.4 \mathrm{~Hz}$ for stably stratified flow (unfilled diamonds) and $f=10.5 \mathrm{~Hz}$ for unstably stratified flow (filled triangles).

The phenomenon of turbulent thermal diffusion can play a crucial role in the dynamics of aerosols in the vicinity of temperature inversion layers in the atmosphere. While atmospheric conditions cannot be exactly reproduced in a laboratory experiment, the ratio of the effective velocity caused by the phenomenon of turbulent thermal diffusion to the terminal fall velocity for $1 \mu \mathrm{m}$ particles in the atmosphere varies in the range of 4-13 (see Table 1), and it is of the same order of magnitude as that in laboratory conditions where it varied in the range of 6-10. In order to obtain the same magnitude of the ratio of the effective velocity to terminal fall velocity as in atmospheric flow conditions, in the laboratory experiments we employed strong mean temperature gradient. We demonstrated that particles in turbulent fluid flow accumulate at the regions with minimum of mean temperature of surrounding fluids due to the phenomenon of turbulent thermal diffusion.

Acknowledgements. We are indebted to F. Busse, H. J. S. Fernando, E. Kit, V. L'vov, A. Tsinober and V. Yakhot for illuminating discussions. We thank A. Krein for his assistance in construction of the experimental set-up and performing the experiments. This work was partially supported by the German-Israeli Project Cooperation (DIP) administrated by the Federal Ministry for Education and Research (BMBF) and by the Israel Science Foundation governed by the Israeli Academy of Science.

Edited by: W.-G. Früh

Reviewed by: J. M. Redondo and two other referees

\section{References}

Aliseda, A., Cartellier, A., Hainaux, F., and Lasheras, J. C.: Effect of preferential concentration on the settling velocity of heavy par- ticles in homogeneous isotropic turbulence, J. Fluid Mech., 468, 77-105, 2002.

Adrian, R. J.: Particle imaging techniques for experimental fluid mechanics, Ann. Rev. Fluid Mech., 23, 261-304, 1991.

Blackadar, A. K.: Turbulence and Diffusion in the Atmosphere, Springer, Berlin, 1997.

Bohren, C. F. and Huffman, D. R.: Absorbtion and Scattering of Light by Small Particles, John Wiley and Sons, New York, 1983.

Borman, G. L. and Ragland, K. W.: Combustion Engineering, MacGraw-Hill, Boston-New-York, 1999.

Buchholz, J., Eidelman, A., Elperin, T., Grünefeld, G., Kleeorin, N., Krein, A., and Rogachevskii, I.: Experimental study of turbulent thermal diffusion in oscillating grids turbulence, Experiments in Fluids, 36, 879-887, 2004.

Crowe, C. T., Sommerfeld, M., and Tsuji, Y.: Multiphase flows with particles and droplets, CRC Press, NY, 1998.

Csanady, G. T.: Turbulent Diffusion in the Environment, Reidel, Dordrecht, 1980.

Eidelman, A., Elperin, T., Kleeorin, N., Krein, A., Rogachevskii, I., Buchholz, J., and Grünefeld, G.: Turbulent thermal diffusion of aerosols in geophysics and in laboratory experiments, Nonlin. Processes Geophys., 11, 343-350, 2004 , mboxhttp://www.nonlin-processes-geophys.net/11/343/2004/.

Eidelman, A., Elperin, T., Kleeorin, N., Rogachevskii, I., and SapirKatiraie, I.: Turbulent thermal diffusion in a multi-fan turbulence generator with imposed mean temperature gradient, Experiments in Fluids, in press, 2006.

Elperin, T., Kleeorin, N., and Rogachevskii, I.: Turbulent thermal diffision of small inertial particles, Phys. Rev. Lett., 76, 224-228, 1996.

Elperin, T., Kleeorin, N., and Rogachevskii, I.: Turbulent barodiffusion, turbulent thermal diffusion and large-scale instability in gases, Phys. Rev. E, 55, 2713-2721, 1997.

Elperin, T., Kleeorin, N., and Rogachevskii, I.: Mechanisms of formation of aerosol and gaseous inhomogeneities in the turbulent atmosphere, Atmos. Res., 53, 117-129, 2000a.

Elperin, T., Kleeorin, N., Rogachevskii, I., and Sokoloff, D.: Turbulent transport of atmospheric aerosols and formation of largescale structures, Phys. Chem. Earth, A25, 797-803, 2000b.

Elperin, T., Kleeorin, N., Rogachevskii, I., and Sokoloff, D.: Meanfield theory for a passive scalar advected by a turbulent velocity field with a random renewal time, Phys. Rev. E, 64, 026304(1-9), 2001.

Fessler, J. R., Kulick, J. D., and Eaton, J. K.: Preferential concentration of heavy particles in a turbulent channel flow, Phys. Fluids, 6, 3742-3749, 1994.

Flagan, R. and Seinfeld, J. H.: Fundamentals of Air Pollution Engineering, Prentice Hall, Englewood Cliffs, 1988.

Glassman, I.: Combustion, Academic Press, San Diego, 1996.

Guibert, P., Durget, M., and Murat, M.: Concentration fields in a confined two-gas mixture and engine in cylinder flow: laser tomography measurements by Mie scattering, Exp. Fluids, 31, 630-642, 2001.

Heywood, J. B.: Internal Combustion Engine Fundamentals, MacGraw-Hill, Boston-New-York, 1988.

Hopfinger, E. J. and Toly, J.-A.: Spatially decaying turbulence and its relation to mixing across density interfaces, J. Fluid Mech., 78, 155-175, 1976.

Jaenicke, R.: Aerosol Physics and Chemistry, Springer, Berlin, 
1987.

Kamimoto, T. and Kobayashi, H.: Combustion processes in diesel engines, Prog. Energy Combust. Sci., 17, 163-189, 1991.

Kit, E., Strang, E. J., and Fernando, H. J. S.: Measurement of turbulence near shear-free density interfaces, J. Fluid Mech., 334, 293-314, 1997.

Landau, L. D. and Lifshits, E. M.: Fluid mechanics, Pergamon, Oxford, 1987

Maxey, M. R.: The gravitational settling of aerosol particles in homogeneous turbulence and random flow field, J. Fluid Mech., 174, 441-465, 1987.

Medina, P., Sánchez, M. A., and Redondo, J. M.: Grid stirred turbulence: applications to the initiation of sediment motion and lift-off studies, Phys. Chem. Earth, B 26, 299-304, 2001.

Ott, S. and Mann, J.: An experimental investigation of the relative diffusion of particle pairs in three-dimensional turbulent flow, $\mathrm{J}$. Fluid Mech., 422, 207-234, 2000.

Pandya, R. V. R. and Mashayek, F.: Turbulent thermal diffusion and barodiffusion of passive scalar and dispersed phase of particles in turbulent flows, Phys. Rev. Lett., 88, 044501(1-4), 2002.

Raffel, M., Willert, C., and Kompenhans, J.: Particle Image Velocimetry, Springer, 1998

Reeks, M. W.: On model equations for particle dispersion in inhomogeneous turbulence, Int. J. Multiphase Flow, 31, 93-114, 2005.
Sánchez, M. A. and Redondo, J. M.: Observations from grid stirred turbulence, Appl. Sci. Res., 59, 243-254, 1998.

Seinfeld, J. H.: Atmospheric Chemistry and Physics of Air Pollution, John Wiley, New York, 1986.

Shaw, R. A.: Particle-turbulence interactions in atmospheric clouds, Ann. Rev. Fluid Mech., 35, 183-227, 2003.

Telford, J. W.: Clouds with turbulence: the role of entrainment, Atmos. Res., 40, 261-282, 1996.

Thompson, S. M. and Turner, J. S.: Mixing across an interface due to turbulence generated by an oscillating grid, J. Fluid Mech., 67, 349-368, 1975.

Turner, J. S.: The influence of molecular diffusivity on turbulent entrainment across a density interface, J. Fluid Mech., 33, 639656, 1968.

Turner, S. T.: Buoyancy Effects in Fluids, Cambridge Univ. Press, Cambridge, 1973.

Twomey, S.: Atmospheric Aerosols, Elsevier, Amsterdam, 1977.

Warhaft, Z.: Passive scalar in turbulent flows, Ann. Rev. Fluid Mech., 32, 203-240, 2000.

Westerweel, J.: Theoretical analysis of the measurement precision of particle image velocimetry, Experiments in Fluids, Suppl., 29, S3-S12, 2000. 